\title{
SOME ENTIRE FUNGTIONS WITH FIXPOINTS OF EVERY ORDER
}

\author{
I. N. BAKER
}

(received 27 July 1959)

\section{Introduction}

In this paper $f(z)$ will always stand for an entire transcendental function of the complex variable $z$. For $p=1,2, \cdots$ the natural iterate $f_{p}(z)$ of $f(z)$ is defined by

$$
f_{1}(z)=f(z), \quad f_{p}(z)=f_{p-1}(f(z))=f\left(f_{p-1}(z)\right) .
$$

These natural iterates are themselves entire transcendental functions; they have been studied by various writers, notably Fatou [3]. References to many papers on iterates will be found in [1].

A fixpoint of $f(z)$ is a zero of $f(z)-z$; more generally a fixpoint of order $p$ of $f(z)$ is a zero of $f_{p}(z)-z$. A fixpoint of order $p$ is said to have order exactly $p$ when it is not a fixpoint of order less than $p$.

The fixpoints are of great importance in the theory of iteration so that a discussion of their existence and distribution is interesting. In [2] it is pointed out that very little is known about the existence of fixpoints of the various orders and a few results are derived in the case where $f(z)$ has order less than $\frac{1}{2}$. Although it is known that any $f(z)$ has fixpoints of arbitrarily high exact order no examples seem to have been given of functions having fixpoints of order exactly $p$ for every natural number $p$. In this paper it is shown that the class $C_{p}$ of functions $\left\{f(z) ; f_{p}(z)\right.$ has finite defect values the sum of whose defects is greater than $\left.\frac{1}{2}\right\}$ has fixpoints of order exactly $p$. The class formed by the intersection of classes $C_{p}, p=1,2, \cdots$ has fixpoints of all exact orders. In particular any function $f(z)$ with a Picard exceptional value is of this type and there are others as shown by Lemma 4. Finally one may conjecture that any $f(z)$ has fixpoints of every exact order from a certain order on.

\section{Preliminary Lemmas}

The following notation will be used (c.f. Nevanlinna [4]):

$$
M_{p}(r)=M\left(f_{p}, r\right)=\max _{|x|=r}\left|f_{p}(z)\right|
$$




$$
\begin{aligned}
& n_{p}(r, a)=n\left(f_{p}, r, a\right)=\text { number of solutions of } f_{p}(z)=a \text { in }|z| \leqq r \\
& N_{p}(r, a)=\int_{0}^{r} \frac{n_{p}(t, a)-n_{p}(0, a)}{t} d t+n_{p}(0, a) \log r \\
& T_{p}(r)=T\left(f_{p}, r\right)=\frac{1}{2 \pi} \int_{0}^{2 \pi}+{ }^{+}\left|f_{p}\left(r e^{i \varphi}\right)\right| d \varphi=m_{p}(r, \infty) \\
& \delta_{p}(a)=1-\varlimsup_{r \rightarrow \infty} \frac{N_{p}(r, a)}{T_{p}(r)}=\varliminf_{r \rightarrow \infty} \frac{m_{p}(r, a)}{T_{p}(r)} .
\end{aligned}
$$

Lemma 1 (Pólya [5]). Let $e(z), g(z)$ and $h(z)$ be entire functions satisfying

(1) $e(z)=g(h(z))$

(2) $h(0)=0$.

There is a constant $c$ independent of $e, g, h$ with

(3) $M(e, r)>M\left[g, c M\left(h, \frac{r}{2}\right)\right]$.

Further it is clear that the condition (2) can be dropped provided (3) is to hold only for all sufficiently great $r$ and this is the form we shall use in the proof of Lemma 3.

Lemma 2 (e.g. Baker [1, p. 124]). If $f(z)$ is an entire function and $k>1$, $a>1$ are constants, then for all sufficiently large $r$ one has

$$
M\left(f, a r^{k}\right)>M^{k}(f, r)
$$

LEMMA 3. If $f(z)$ has an exceptional value $b$ (taken only a finite number $k$ of times) then $b$ is a value of defect one for $f_{p}(z), p=1,2, \cdots$.

PROoF: Let the roots of $f(z)=b$ be $d_{1}, d_{2}, \cdots d_{k}$. The roots of $f_{p}(z)=b$ are the roots of $f_{p-1}(z)=d_{i}, i=1,2, \cdots, k$. We assume they are counted according to the usual multiplicities so that

$$
n_{p}(r, b)=\sum_{i=1}^{k} n_{p-1}\left(r, d_{i}\right)
$$

and

$$
N_{p}(r, b)=\sum_{i=1}^{k} N_{p-1}\left(r, d_{i}\right) \leqq k T_{p-1}(r)+O(1)
$$

by the first fundamental theorem [4].

Now $[4$, p. 220]

$$
\begin{aligned}
T_{p}(r) & \geqq \frac{1}{3} \log M_{p}\left(\frac{r}{2}\right) \\
& \geqq \frac{1}{3} \log M_{p-1}\left(c M_{1}\left(f, \frac{r}{4}\right)\right) \text { by Lemma } 1,
\end{aligned}
$$


and however small $\varepsilon>0$ is, this becomes greater than

$$
\frac{1}{3} \log M_{p-1}\left(r^{(3 k+1) / 8}\right)
$$

which by Lemma 2 is greater than

$$
\frac{1}{3} \log M_{p-1}^{3 k / \varepsilon}(r) \geqq \frac{k}{\varepsilon} T_{p-1}(r),
$$

all of these inequalities being understood to hold only for sufficiently large $r$. Then from (5), (6)

$$
\varlimsup_{r \rightarrow \infty} \frac{N_{p}(r, b)}{T_{p}(r)} \leqq \varepsilon \text { and hence } \delta_{p}(b)=1 .
$$

LEMMA 4. There are functions $f(z)$ other than those of Lemma 3 such that a value $b$ is of defect one for $f_{p}(z), p=1,2, \cdots$

Proof: Consider a function

$$
f(z)=b+e^{e^{z}} h(z), \quad b>0
$$

where $h(z)$ is a function of order 1 with the properties:

$$
\begin{gathered}
M(h, r)=h(r)>0 \\
\exp \left(\frac{r}{2}\right)<h(r)<e^{r} \text { for large } r \\
h(z) \text { has an infinity of zeros. }
\end{gathered}
$$

We could take $h(z)=\sinh z$ but the proof is just as simple with general $h(z)$. We see that

$$
\begin{aligned}
M(f, r) & =f(r) \\
M\left(f_{p}, r\right) & =f_{p}(r)
\end{aligned}
$$

and

$$
\exp \exp r<f(r)<\exp (2 \exp r)
$$

all hold for large $r$. Now

$$
f_{p}(z)=b+\left\{\exp \exp f_{p-1}(z)\right\} h\left(f_{p-1}(z)\right)
$$

and

$$
\begin{aligned}
& N_{p}(r, b)=N\left(h\left(f_{p-1}\right), r\right) \leqq T\left(h\left(f_{p-1}\right), r\right)<\log h\left(f_{p-1}(r)\right), \\
& N_{p}(r, b)<f_{p-1}(r) .
\end{aligned}
$$

On the other hand $[4$, p. 220]:

$$
T_{p}(r)=T\left(f_{p}, r\right)>\frac{1}{3} \log f_{p}\left(\frac{r}{2}\right)>\frac{1}{3} \log f_{p-1}\left\{h_{2}\left(\frac{r}{2}\right)\right\}
$$


from (9), (11),

$$
h_{2}\left(\frac{r}{2}\right)>\exp \left(\frac{1}{2} h\left(\frac{r}{2}\right)\right)>\exp \left(r^{2}\right)
$$

by Liouville's theorem and

$$
\begin{aligned}
& f\left(h_{2}\left(\frac{r}{2}\right)\right)>f\left(\exp \left(r^{2}\right)\right)>\exp \exp \exp \left(r^{2}\right)>\exp \exp \left(4 e^{r}\right) \\
& =\exp \left\{\left(\exp 2 e^{r}\right)^{2}\right\}>\exp \left\{f^{2}(r)\right\} .
\end{aligned}
$$

By induction

$$
f_{p-1}\left(h_{2}\left(\frac{r}{2}\right)\right)>\exp \left\{f_{p-1}^{2}(r)\right\}
$$

and from (13)

$$
T_{p}(r)>\frac{1}{3} f_{p-1}^{2}(r)
$$

Together with (12) this gives

$$
\frac{N_{p}(r, b)}{T_{p}(r)}<\frac{3}{f_{p-1}(r)} \rightarrow 0 \text { as } r \rightarrow \infty .
$$

Thus $\delta_{p}(b)=1$. All the inequalities above are supposed to hold only for sufficiently large $r$.

\section{The Results on Fixpoints}

TheOREM. Suppose $f(z)$ has defect values $b_{i}, i=1,2, \cdots, k$ so that

$$
\sum_{i=1}^{k} \delta_{p}\left(b_{i}\right)=\frac{1}{2}+d ; \quad d>0, \quad b_{i} \neq \infty .
$$

Then $f(z)$ has fixpoints of order exactly $p$.

If $m_{p}\left(r, b_{i}\right)$ is the "Schmiegungsfunktion" defined by

$$
m_{p}\left(r, b_{i}\right)=\frac{1}{2 \pi} \int_{0}^{2 \pi} \log \left|\frac{1}{f_{p}\left(r e^{i \varphi}\right)-b_{i}}\right| \mathrm{d} \varphi
$$

we have

$$
\sum_{i=1}^{k} m_{p}\left(r, b_{i}\right)>\frac{1+d}{2} T_{p}(r)=\frac{1+d}{2} T\left(f_{p}, r\right)
$$

for all sufficiently large $r$. Denote by $m_{p}^{\prime}, m_{p}^{\prime \prime}, T_{p}^{\prime}, T_{p}^{\prime \prime}$ the Schmiegung and characteristic functions for $f_{p}^{\prime}(z)$ and $f_{p}^{\prime \prime}(z)$ respectively and by $\bar{m}_{p}, T_{p}$ the functions for $f_{p}(z)-z$.

In his discussion of the second fundamental theorem Ullrich $[6$, p. 598 equation (20)] has proved a result which we write as 


$$
m_{p}^{\prime}(r, 0) \geqq \sum_{i=1}^{k} m_{p}\left(r, b_{i}\right)-O\left[\log \left(r T_{p}(r)\right)\right]
$$

where the symbol $(E)$ means that the given estimate of the remainder term holds with the possible exception of a set of $r$-intervals whose total length is finite. Thus in our case

$$
m_{p}^{\prime}(r, 0) \geqq \frac{1+d}{2} T_{p}(r)-O\left[\log \left(r T_{p}(r)\right)\right]
$$

We note that

$$
\begin{aligned}
T_{p}^{\prime}(r) & =m_{p}^{\prime}(r, \infty)+O(1) \\
& \leqq m_{p}(r, \infty)+m\left(\frac{f_{p}^{\prime}}{f_{p}}, r, \infty\right)+O(1) \\
& =T_{p}(r)+O\left[\log \left(r T_{p}(r)\right)\right]
\end{aligned}
$$

by the theorem of the logarithmic derivative [e.g. 7 p. 594]. From the second fundamental theorem and (16):

$$
\begin{aligned}
m_{p}^{\prime}(r, 0)+m_{p}^{\prime}(r, 1) & \leqq T_{p}^{\prime}(r)+O\left[\log \left(r T_{p}^{\prime}(r)\right)\right] \\
& \leqq T_{p}(r)+O\left[\log \left(r T_{p}(r)\right)\right]
\end{aligned}
$$

Applying the result of Ullrich used in (14) but this time to the function $f(z)-z$ and its derivative we obtain

$$
\bar{m}_{p}(r, 0) \leqq m_{p}^{\prime}(r, 1)+O\left[\log r T_{p}(r)\right]
$$

and using (15), (17):

$$
\begin{aligned}
\bar{m}_{p}(r, 0) & \leqq m_{p}^{\prime}(r, 1)+O\left[\log \left(r T_{p}(r)\right)\right] \\
& \leqq\left(\frac{1-d}{2}\right) T_{p}(r)+O\left[\log \left(r T_{p}(r)\right)\right]
\end{aligned}
$$

Using the first fundamental theorem and $T_{p}^{\prime}(r) \simeq T_{p}(r)$ it follows that

$$
\bar{N}_{p}(r, 0) \geqq\left(\frac{1+d}{2}\right) T_{p}(r)-O\left[\log \left(r T_{p}(r)\right)\right]
$$

Now by a further application of the result of (14) and (15):

$$
\begin{aligned}
m_{p}^{\prime \prime}(r, 0) & \geqq m_{p}^{\prime}(r, 0)-O\left[\log \left(r T_{p}^{\prime}(r)\right)\right] \\
& \geqq \frac{1+d}{2} T_{p}(r)-O\left[\log r T_{p}(r)\right]
\end{aligned}
$$

and

$$
N_{p}^{\prime \prime}(r, 0) \leqq T_{p}^{\prime \prime}(r)-\left(\frac{1+d}{2}\right) T_{p}(r)+O\left[\log \left(r T_{p}(r)\right)\right]
$$


while from (16):

$$
T_{p}^{\prime \prime}(r) \leqq T_{p}(r)+O[\log (r T(r))]
$$

which reduces (19) to

$$
N_{p}^{\prime \prime}(r, 0) \leqq\left(\frac{1-d}{2}\right) T_{p}(r)+O\left[\log \left(r T_{p}(r)\right)\right]
$$

Thus from (18), (19):

$$
\frac{1}{2}\left\{N_{p}(r, 0)-N_{p}^{\prime \prime}(r, 0)\right\} \geqq \frac{d}{2} T_{p}(r)-O\left[\log \left(r T_{p}(r)\right)\right]
$$

and for some values of $r$ the quantity on the left hand side of (20) will take large values of the same order as $d T_{p}(r) / 2$.

A $k$-fold $(k \geqq 1)$ zero of $f_{p}(z)-z$ is counted $k$ times in $\bar{N}_{p}(r, 0)$ but only $\operatorname{Max}\{0, k-2\}$ times in $N_{p}^{\prime \prime}$ so that the left hand side of $(20)$ is not greater than

$$
N_{+}(r, 0)=\int_{0}^{r} \frac{n_{+}(t, 0)-n_{+}(0,0)}{t} d t+n_{+}(0,0) \log r
$$

where $n_{+}(t, 0)$ counts the number of different solutions of $f_{p}(z)=z$ in $|z| \leqq t$. For all sufficiently large $r$

$$
\sum_{j=1}^{p-1} N_{j}(r, 0) \leqq \sum_{j=1}^{p-1} T_{j}(r, 0)+O(1)
$$

and by Lemma 1 (as applied in Lemma 3) this right hand side of (21) is $o\left(T_{p}(r, 0)\right.$ for large $r$. The left hand side of $(21)$ is an upper bound for the contribution of fixpoints of orders less than $p$ to $N_{+}(r, 0)$ since each of them is counted at least once there. Thus from (20) the counting function of different fixpoints of order $p$ is $>d T_{p}(r) / 3$ for some arbitrarily large values of $r$, while from (21) this is not caused by the fixpoints which are of exact order less than $p$. It follows that the fixpoints of exact order $p$ have a counting function $\tilde{N}(r)$ which satisfies

$$
\varlimsup_{r \rightarrow \infty} \frac{\tilde{N}(r)}{T(r)}>0
$$

and that a great many such fixpoints exist.

Application: The functions of Lemma 3 and 4 afford examples of functions which have fixpoints of exact order $p$ for all natural numbers $p$. 
Note: Professor W. K. Hayman has pointed out that the constant $\frac{1}{2}+d$ in the above theorem can be replaced by $d$ alone if $d>0$, i.e. it is sufficient to suppose that $f_{p}(z)$ has some defective value $b$. One has only to apply Nevanlinna's theory to the function $\left(f_{p}(z)-z\right) /\left(f_{p}(z)-b\right)$.

\section{References}

[1] Baker, I. N., Zusammensetzungen ganzer Funktionen. Math. Zeit. 69, 121-163 (1958).

[2] Baker, I. N., Fixpoints and iterates of entire functions. Math. Zeit. 71, 146-153 (1959).

[3] Fatou, P., Sur l'itération des fonctions transcendantes entières. Acta math. 47, 337-370 (1926)

[4] Nevanlinna, R., Eindeutige analytische Funktionen. 2 Aufl. Berlin, Springer 1953.

[6] Polya, G., On an integral function of an integral function. J. London Math. Soc. 1, 12-15 (1926).

[6] Ullrich, E., Sitzungsberichte preuss. Akad. M. P. Klasse. 1929 (592-608).

Imperial College of Science and Technology, London 